\title{
Competencia conceptual y de procedimiento: comprensión de la propiedad conmutativa de la adición y estrategias de solución
}

\author{
VICENTE BERMEJO, PURIFICACIÓN RODRÍGUEZZ \\ Universidad Complutense de Madrid', E. U. de Profesorado de EGB de Segovia ${ }^{2}$
}

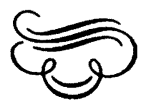

Resumen

En esta investigación se analizan los factores implicados en la adquisición de la estrategia aditiva de contar desde el sumando mayor y su relación con el conocimiento de la propiedad conmutativa de la suma. Tres grupos de niños con edades comprendidas entre los 5 y los 8 años resuelven tres tareas (resolver sumas, comparar sumas y encontrar el sumando desconocido), en dos condiciones experimentales (presencial ausencia del resultado) y con cuatro tipos de sumandos $(1+N$; círculos + guarismos; bechos numéricos; $y$ bechos numéricos superiores a la decena). En general, los resultados muestran que los factores grupo, tarea, presencialausencia del resultado y tipo de sumandos influyen en el rendimiento de los niños. Asimismo, el análisis de las estrategias y errores nos ba permitido constatar la existencia de diferencias evolutivas entre los grupos. Por último, nuestros datos ponen de manifiesto que las estrategias aditivas de contar desde el mayor se acompañan de un cierto éxito en las tareas de conmutatividad y que el fracaso en la tarea de sumar no implica fracaso igualmente en las tareas de conmutatividad.

\section{Abstract}

In this research authors analyzed the factors involved in the acquisition of the additive strategy of counting from the larger addend, and its relationship with the knowledge of the commutative law of addition. Three groups of children, with ages between 5 and 8 years, solved three tasks (i.e., solve additions; compare sums; and find the unknown addend), in two experimental conditions (i.e, presencel absence of the result) and with four types of addends (i.e., $1+N$; circles + numbers; numerical facts; and numerical facts greater than ten). In general, the results showed that the factors of group, task, presencel absence of the result, and kind of addend affected children's level of attainment. Moreover; the analysis of the strategies and errors let us to verify the existence of developmental differences among the groups. Finally, our data pointed out that the additive strategies in which children start to count from the larger addend were linked to a certain success in the commutativity tasks, and that the failure in the additive task didn't involve a similar failure in the commutativity tasks.

Correspondencia con autores: ${ }^{1}$ Departamento de Psicología Evolutiva ỵ de la Educación. Campus de Somosaguas, $28023 \mathrm{Madrid}^{2}{ }^{2}$ Este estudio recoge algunas de las ideas contenidas en la tesis doctoral de P. Rodríguez. 


\section{INTRODUCCION}

Recientemente, diversos autores (Hiebert y Lefevre, 1986; Silver, 1986; Vanlehn, 1986; etc.) que trabajan en el ámbito de las matemáticas han insistido en la necesidad de distinguir entre conocimiento conceptual y de procedimiento. El primero ha sido caracterizado como el conocimiento de los principios y reglas y el segundo como el conocimiento de las habilidades o estrategias. Byrnes y Wasik (1991) presentan tres argumentos para justificar la necesidad de distinguir entre estos dos tipos de conocimiento. El primero hace referencia a que, desde el punto de vista lógico, el conocimiento de procedimiento no es reducible al conocimiento conceptual. El segundo se relaciona con el hecho de que estos dos tipos de conocimiento tienen diferentes funciones cognitivas, de modo que los conceptos imponen orden y organización sobre las experiencias formando categorías o relacionando sucesos causalmente en el tiempo o en el espacio, mientras que los procedimientos son medios para alcanzar un fin. El último argumento es de talante empírico y permite explicar aquellos casos en los que los sujetos presentan altos niveles de conocimiento conceptual y carencia de conocimiento de procedimiento, o viceversa. Por su parte, Hiebert y Lefevre (1986) añaden que la distinción entre estas dos clases de conocimiento proporcionan un marco adecuado para examinar cuestiones relativas al proceso de adquisición del conocimiento.

No obstante, uno de los retos más importantes que tiene planteada la investigación consiste en determinar cómo se producen las conexiones entre conocimiento conceptual y de procedimiento o, dicho en otras palabras, cómo se relacionan los conceptos y los procedimientos. A este respecto, Carpenter (1986) apunta, por un lado, que los avances en el conocimiento conceptual son difíciles de identificar y de medir directamente y, en todo caso, lo que hacemos es inferirlo a partir de la observación de ciertos procedimientos para los que se supone que constituye un prerrequisito. Por otro lado, los conceptos son aplicados en dominios muy específicos antes de ser generalizados más ampliamente. En esta misma línea, Baroody y Ginsburg (1986) indican que los procedimientos utilizados por los niños en la resolución de una tarea no siempre reflejan su nivel de competencia conceptual real.

Otra de las dificultades con que se encuentra la investigación en esta área es averiguar hasta qué punto los avances en el conocimiento de procedimiento ocurren como resultado de los avances en el conocimiento conceptual. Aquí es donde las posturas enfrentadas suelen ser más frecuentes. Así, por un lado, Carpenter (1986) indica que resulta incorrecto afirmar que el conocimiento conceptual sea de algún modo innecesario para producir avances en el conocimiento de procedimiento. En este sentido, aunque los niños pueden llegar a utilizar procedimientos sin vincularlos con una forma de conocimiento conceptual, cuando algunas invenciones se producen exclusivamente en relación con el conocimiento de procedimiento, a menudo conducen a error. Por otro, Baroody y Ginsburg (1986) consideran que el desarrollo del conocimiento conceptual no asegura la adquisición de procedimientos relacionados con dicho conocimiento y que, asimismo, la construcción de procedimientos avanzados puede deberse tanto a la adquisición del conocimiento conceptual subyacente como a un intento de reducir las demandas de procesamiento cognitivo de la tarea.

Este debate se ha extendido a numerosos aspectos de la aritmética elemental y así, en relación con las estrategias aditivas, por ejemplo, no está claro hasta qué punto la utilización por parte del niño de estrategias de solución sofisticadas implica un mayor nivel conceptual. Más específicamente en la estrategia de contar desde el sumando mayor, algunos autores (Briars y Larkin, 1984; Carpenter, 1986; Riley, Greeno y Heller, 1983) señalan que el conocimiento de la conmutatividad constituye una condición necesaria para la comprensión y uso de esta 
estrategia. En una línea diferente, Baroody y colaboradores (Baroody y Gannon, 1984; Baroody y Ginsburg, 1986; Baroody, Ginsburg y Waxman, 1983) sugieren que el conocimiento de la propiedad conmutativa no se liga necesariamente con el uso de estrategias aditivas de contar a partir del sumando mayor. Posición esta última compartida por Resnick (1983), puesto que sugiere que eșta estrategia no deriva sistemáticamente. del descubrimiento dé la conmutatividad, sino que resulta de la práctica en la suma. En efecto, en un programa de ordenador (HPM) desarrollado por Resnick y Neches.(1984) para explicar el descubrit miento de los niños del procedimiento de contar a partir del mayor, atribuyen la aparición del mismo a la presencia de un esquema de etiquetado de orden indiferente y de dos heurísticos: resultado aún disponible y resultado no modificado (para una mayor descripción ver Rodríguez, en prensa).

Por lo que se refiere a la conmutatividad, tampoco resulta claro si la adquisición de la misma se produce como resultado de un conocimiento más sofisticado de la adición o como resultado de la práctica en la rẻsolución de tareas aditivas. A este respecto y de acuerdo con la primera posición, el conocimiento de la conmutatividad se vincula con una comprensión implícita de las relaciones parte-todo (Briarś y Larkin, 1984; Resnick y Neches, 1984; Riley et al., 1983) o en términos de Weaver (1982) con la concepción binaria de la suma. La segunda se halla en la línea dé Baroody, ya que afirma que los niños son capaces de descubrir esta propiedad por medios informales, sin necesidad de una concepción binaria de la suma, siempre y cuando se les dé la oportunidad de comparar los resultados de pares de algoritmos conmutados.

El presente trabajo persigue un doble objetivo. Por una parte, analizar los factores implicados en la adquisición de las estrategias aditivas consistentes en contar desde el sumando mayor (contar todó empezando por el mayor y contar a partir del mayor) y su vinculación con el conocimiento de la propiedad conmutativa. Por otra, se pretende profundizar en la relación existente entre el nivel de comprensión de la operaçión aditiva y el nivel adquirido con respecto a la propiedad conmutativa. Para ello hemos seguido un diseño factorial mixto 3 (grupo) x 2 (presencia/ausencia del resultado) x 3 (tareas) $\times 4$ (tipo de sumandos), planteando las siguientes hipótesis generales:

Hipótesis 1: Se observarán diferencias evolutivas entre los grupos en cuanto al nivel de rendimiento en función del tipo de tareas, el tipo de sumandos y la presencia/ausencia del resultado.

Hipótesis 2: Las estrategias y errores cometidos por los niños en las distintas tareas variarán en función de la edad, presencia/ausencia del resultado, la tärea y el tipo de sumandos.

Hipótesis 3: La utilización de estrategias consistentes en contar empezando por el mayor en la resolución de las tareas aditivas supone cierta competencia en la propiedad conmutativa.

A diferencia de otros autores (Baroody y Ginsburg, 1986; Resnick y Neches, 1984), que señalan que los niños inventan estas estrategias, independientemente de su conocimiento sobre la conmutatividad, nos proponemos demostrar, de acuerdo con Carpenter (1986), que el conocimiento del procedimiento de contar desde el sumando mayor no puede desvincularse de un cierto conocimiento conceptual de la propiedad conmutativa de la suma.

Hipótesis 4: El fracaso del niño en ciertas tareas aditivas no acompaña necesariamente a un fracaso en las tareas de conmutatividad, sino que en ciertas ocasiones estas últimas pudieran resultar más sencillas.

En otras palabras, ciertas tareas aditivas como, por ejemplo, hallar el sumando inicial, pueden resultar muy complejas para los niños, mientras que no ocurre así en las tareas de conmutatividad, ya que el niño puede extraer la respuesta mediante una simple estrategia perceptiva comprobando que en los dos algoritmos que ha de comparar o completar se encuentran los mismos números. 


\section{METTODO}

\section{Sujetos}

Participaron en el estudio un total de 72 niños elegidos al azar, pertenecientes a un colegio madrileño de nivel socio-económico medio, distribuidos en tres grupos de 24 sujetos cada uno. El grupo I estaba formado por niños de segundo de preescolar, cuyas edades oscilaban entre los 5-6 años (M: 5;6); en el grupo II se incluían niñõos de $10^{\circ}$ de EGB con edades comprendidas entre los 6-7 años (M: 6;4); por último, el grupo III estaba compuesto por niños de $2 .^{\circ}$ de EGB con edades de 7-8 años (M: 7;6).

\section{Material y procedimiento experimental}

El material estaba constituido por cuadernillos, que contenían las pruebas, y un lápiz para anotar las respuestas.

En cuànto al procedimiento experimental, las tareas eran leídas por el experimentador en voz alta. Sin limitaciones de tiempo, los niños debían responder en unos casos verbalmente y en otros de modo escrito, en función del tipo de tarea que debían resolver (producción o verificación), llevándose a cabo una filmación en vídeo de todo el proceso de ejecución. Además, las pruebas fueron presentadas de modo individual a los niños en dos momentos distintos separados por una semana. En la primera aplicación la mitad de los sujetos de los distintos grupos resolvían las tareas en las que estaba presente el resultado, mientras que la otra mitad lo hacía con aquellas en las que el resultado se encontraba ausente. En la segunda ocasión se invirtieron los términos. De este modo, se evitaba que la duración de las pruebas fuera excesiva, ocasionando fatiga en los niños y controlando al mismo tiempo el posible efecto del aprendizaje de unas pruebas sobre otras.

Las tareas propuestas eran de tres tipos: (1) resolución de sumas, (2) comparación de sumas y (3) encontrar el sumando desconocido. La primera y la tercera eran tareas de producción, en las que el sujeto debía construir la respuesta; mientras que la segunda constituía una tarea de verificación en la que simplemente tenía que indicar si era verdadera o falsa la situación propuesta y explicar las razones que le llevaban a tomar esa decisión.

La tarea de resolución de sumas se presentaba bajo la forma de problemas verbales de cambio, pero con el algoritmo ya escrito, de modo que el niño tenía que encontrar el elemento desconocido. Por ejemplo:

Al principio Juan tenía estos caramelos. María le dio éstos. ¿Cuántos caramelos tiene abora Juan en total?

$$
1+13
$$

La tarea de encontrar el sumando desconocido era una prueba de conmutatividad. Se presentaban dos algoritmos aditivos, de modo que uno estaba completo mientras que en el otro se desconocía uno de los sumandos. Lo que se pedía al niño era que determinase el elemento que faltaba para tener exactamente lo mismo que había en el otro algoritmo, obteniéndose la solución correcta al poner en relación ambos algoritmos. Por ejemplo:

César tiene estas canicas y Tomás tiene éstas. ¿Cuántas canicas necesita Tomás para tener las mismas que César. 
Finalmente, la tarea de comparación de sumas consistía en una prueba de conmutatividad en la que el niño debía comparar pares de algoritmos conmutados, para determinar si ambos eran o no equivalentes. Por ejemplo:

Luis tiene estos caramelos y Pedro tiene éstos. ¿Tienen los dos el mismo número de caramelos?

$1+16$

$16+1$

En esta última tarea, cuando el resultado estaba ausente, se pedía a los niños que resolviesen ambas cuentas en el sumando «hechos numéricos», una vez contestadas las preguntas sobre conmutatividad en este mismo sumando. El objetivo era doble: por una parte, comprobar si los sujetos que admitían la igualdad de los algoritmos aplicaban este conocimiento a la resolución de las tareas aditivas al aparecer los algoritmos conmutados; por otra parte, se trataba de presentar cantidades familiares para los niños de preescolar y fácilmente representables con los dedos. Adèmás, únicamente solicitábamos la resolución del algoritmo aditivo en este sumando, para no alargar la prueba excesivamente y provocar cansancio en los niños.

En cuanto a las cantidades, se utilizaron cuatro tipos de sumandos en cada tarea: $1+\mathrm{N}$ (p.e., $1+8$ ), círculos más guarismos (p.e., $000000+13$ ), hechos numéricos (p.e., $3+4)$ y hechos numéricos que superaban la decena $(5+12)$. Estos sumandos eran los mismos en ambas partes del experimento.

Finalmente, en cada tarea se proponían tres ensayos para cada tipo de sumando, contrabalanceándose tanto el orden de presentación de las tareas como el de los sumandos.

\section{ANAUISIS Y DISCUSION DE RESULTADOS}

\section{Análisis cuantitativo de los resultados}

El análisis de varianza mixto 3 (grupo: G. I vs. G. II vs. G. III) x 2 (resultado: presencia vs. ausencia del resultado) $\times 3$ (tareas: resolver sumas vs. comparar sumas conmutativas vs. encontrar el sumando desconocido) x 4 (sumandos: $1+\mathrm{N}$ vs. círculos + guarismos vs. hechos numéricos vs. hechos numéricos superiores a la decena) con medidas repetidas en los tres últimos factores y llevado a cabo mediante el programa BMDP (2V), muestra, por un lado, que son significativos los efectos principales de los factores grupo $\left(\mathrm{F}_{2,69}=19.88, \mathrm{p}<0.0000\right)$, tipo de tarea $\left(\mathrm{F}_{2,139}=10.46, \mathrm{p}<0.0001\right)$, presencia/ausencia del resultado $\left(\mathrm{F}_{1,69}=80.32, \mathrm{p}<0.0000\right)$ y el tipo de sumandos $\left(\mathrm{F}_{3,207}=7, \mathrm{p}<0.0002\right)$. Por tanto, tal como podemos observar en la Tabla I, existen diferencias significativas entre los grupos, correspondiendo las medias más altas al grupo de los mayores, siguiéndoles a continuación los de $1 .^{\circ}$ de EGB y, por último, los preescolares. Además, en todos los grupos de edad, las puntuaciones resultan superiores en la condición de ausencia del resultado. Asimismo, el tipo de tarea propuesta y el tipo de sumandos dan lugar a respuestas significativamente distintas.

Por otro lado, el análisis de varianza indica que son significativas las siguientes interacciones: grupo $\mathrm{x}$ presencia del resultado $\left(\mathrm{F}_{2,69}=3.82, \mathrm{p}<0.02\right)$, grupo $\mathrm{x}$ tarea $\left(\mathrm{F}_{4,138}=2.57, \mathrm{p}<0.04\right)$, grupo $\mathrm{x}$ tipo de sumandos $\left(\mathrm{F}_{6,27}=4.28, \mathrm{p}<0.0004\right)$, tarea $\mathrm{x}$ tipo de sumandos $\left(\mathrm{F}_{6,414}=18.49, \mathrm{p}<0.0000\right)$, grupo $\mathrm{x}$ tipo de tarea $\mathrm{x}$ tipo de sumandos $\left(\mathrm{F}_{12,414}=2.16, \mathrm{p}<0.01\right)$, presencia del resultado $\mathrm{x}$ tipo de sumandos $\mathrm{x}$ grupo $\left(\mathrm{F}_{6,207}=2.58, \mathrm{p}<0.01\right)$, presencia del resultado $\mathrm{x}$ tipo de tarea $\mathrm{x}$ tipo de suman$\operatorname{dos}\left(\mathrm{F}_{6,414}=4.07, \mathrm{p}<0.0006\right)$ resultado $\mathrm{x}$ tipo de tarea $\mathrm{x}$ tipo de sumandos $\mathrm{x}$ grupo $\left(\mathrm{F}_{12,414}=3.13, \mathrm{p}<0.0003\right)$. El análisis de las interacciones se lleva a cabo mediante las comparaciones de interacción con la prueba de Scheffé. A este respecto, analizamos únicamente las interacciones triples descartando las dobles y la cuádruple para evitar repeticiones innecesarias. 


\section{8}

TABLA I

Medias y desviaciones típicas, entre paréntesis, del ANOVA

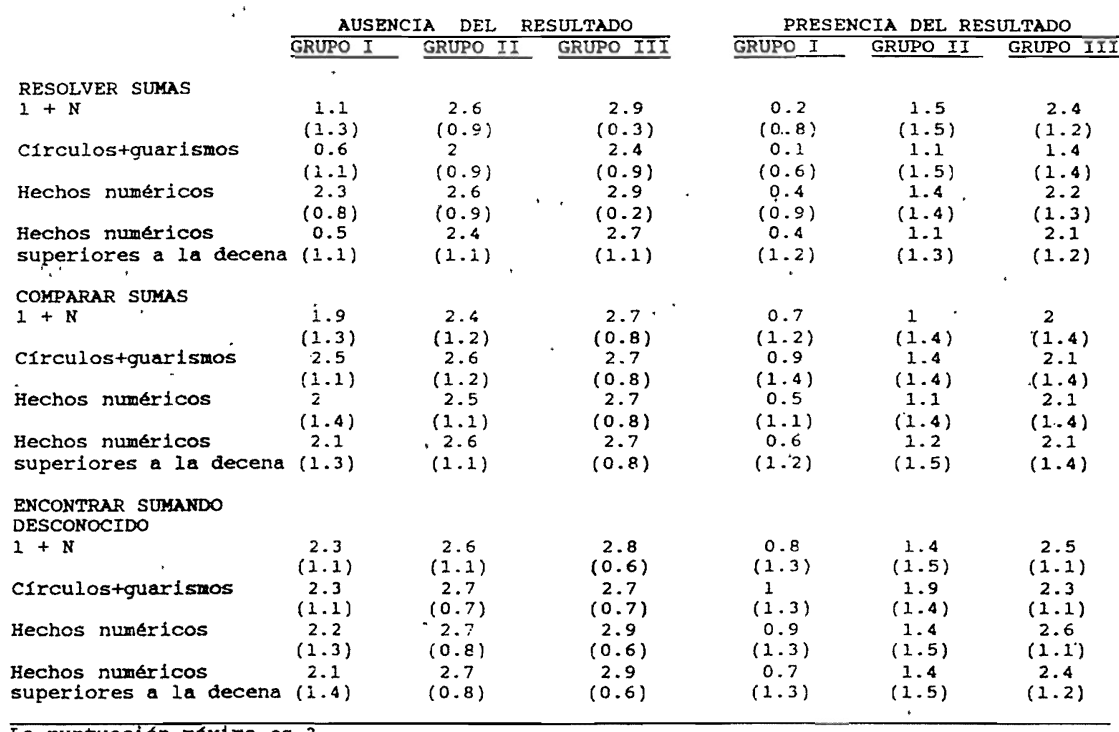

\section{Análisis de las relaciones entre los factores grupo, tipo de tarea. y tipo de sumandos}

Respecto a la interacción grupo $\mathrm{x}$ tipo de tarea $\mathrm{x}$ tipo de sumandos efectuamos dos contrastes de interacción: (a) entre los distintos niveles de los factores grupo y tipo de tarea, asignando el mismo peso a todos los sumandos, y (b) entre los distintos niveles de los factores grupo y tarea en cada uno de los sumandos. Los resultados en el primer tipo de comparaciones ponen de manifiesto la existencia de diferencias significativas en las tareas de resolver sumas y comparar sumas entre el $G$. I y el G. II $\left(\mathrm{F}_{1,414}=29.2\right.$, p $\left.<0.01\right)$, el G. I y el G. III $\left(\mathrm{F}_{1,414}=21.98, \mathrm{p}<0.01\right)$, pero no entre los grupos de EGB en estas mismas tareas. Los contrastes de interacción de las tareas de resolver sumas y encontrar el sumando desconocido muestran que resultan significativas las diferencias entre el G. I y el G. II $\left(\mathrm{F}_{1,414}=19.81, \mathrm{p}<0.01\right)$ y entre el G. I y $\mathrm{G}$. III $\left(\mathrm{F}_{1,414}=16.43, \mathrm{p}<001\right)$ (Figura 1$)$.

Finalmente, aun cuando no resultan significativas las diferencias entre las tareas conmutatividad con respecto a los grupos, se observa en todos ellos un nivel de ejecución ligeramente superior en la tarea de encontrar el sumando desconocido (Figura 1). Además, en presencia del resultado los niños de todos los grupos ejecutan peor la tarea de comparar sumas que la de encontrar el sumando desconocido, a pesar de que esta última es una tarea de producción y no se presenta bajo la forma canónica. Este dato es asimismo confirmado en los resultados procedéntes de la aplicación de la Q de Yule para ver el grado de asociación entre las tareas de conmutatividad y del log-odds para verificar si se trata de una asociación perfecta u ordenada. Así, se observa que en la condición presencia del resultado, la tarea de encontrar el sumando desconocido se desarrolla antes en todos los grupos y en todos los sumandos, salvo en el G. I en el sumando $1+\mathrm{N}$. En ausencia del resultado se mantiene esta pauta gene- 
ral en el G. III y en los sumandos círculos + guarismo y hechos numéricos en el G. II; en el G. I no existe asociación entre estas tareas en ninguno de los sumandos (para más detalles ver Rodríguez, en prensa).

FiguRA 1

Medias correspondientes a la interacción A (grupo) $\times C$ (tarea) $\times D$ (sumandos)

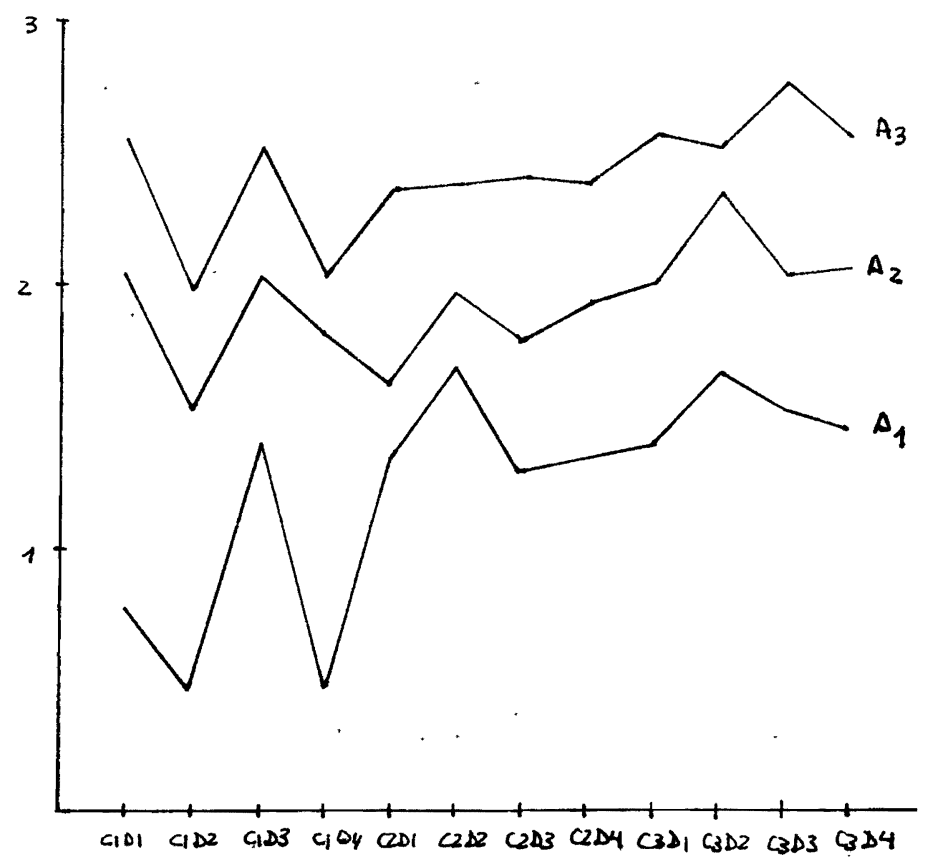

Por lo que hace referencia a los contrastes de interacción entre los distintos niveles de los factores grupo y tareas en cada uno de los sumandos, hallamos diferencias significativas entre los grupos I y II $\left(F_{1,414}=9.85, \mathrm{p}<0.01\right)$ y I-III $\left(\mathrm{F}_{1,414}=10.88\right.$, $\mathrm{p}<0.01)$ en las tareas de resolver sumas-encontrar el sumando desconocido en el sumando $1+\mathrm{N}$; entre los.grupos I-II $\left(\mathrm{F}_{1,414}=11.96\right.$; $\left.\mathrm{p}<0.01\right)$ y I-III $\left(\mathrm{F}_{1,414}=9.36\right.$, $\mathrm{p}<0.01)$ en las tareas de resolver sumas-comparar sumas en el sumando círculos + guarismos y también para este mismo sumando entre los grupos I-III $\left(\mathrm{F}_{1,414}=6.67\right.$, $\mathrm{p}<0.01$ ) en las tareas resolver sumas-encontrar el sumando desconocido. En el sumando hechos numéricos superiores a la decena, alcanzan la significatividad las diferencias entre los grupos I-II $\left(\mathrm{F}_{1,414}=9.85, \mathrm{p}<0.01\right)$ y I-III $\left(\mathrm{F}_{1,414}=8.41, \mathrm{p}<0.01\right)$ en las tareas resolver sumas-comparar sumas, y entre los grupos I-II $\left(\mathrm{F}_{1,414}=7.52, \mathrm{p}<0.01\right)$ y I-IIII $\left(\mathrm{F}_{1,414}=4.95, \mathrm{p}<0.5\right)$ en las tareas resolver sumas-encontrar el sumando desconocido.

\section{Análisis de las relaciones entre los factores presencia/ausencia del resultado, tarea y sumandos}

En cuanto a la interacción presencia del resultado $\mathrm{x}$ tarea $\mathrm{x}$ tipo de sümandos, llevamos a cabo, por un lado, comparaciones de interacción entre los distintos niveles del factor tipo de tarea, asignando el mismo peso a los sumandos, en el factor presencia del resultado. Los resultados apuntan diferencias significativas únicamente entre la tarea de resolver sumas y comparar sumas $\left(\mathrm{F}_{1,414}=8.55, \mathrm{p}<0.01\right)$. Estas diferencias son imputables a la tarea de comparar sumas, ya que aun cuando esta tarea resulta más sencilla, tanto en presencia como en ausencia del resultado, las dis- 
tancias entre las puntuaciones medias en esta prueba en ambas condiciones son mayores que en la de resolver sumas (Figura 2).

FIGURA 2

Medias correspondientes a la interacción $B$ (presencialausencia del resultado) $\times C$ (tarea) $\times D$ (sumandos)

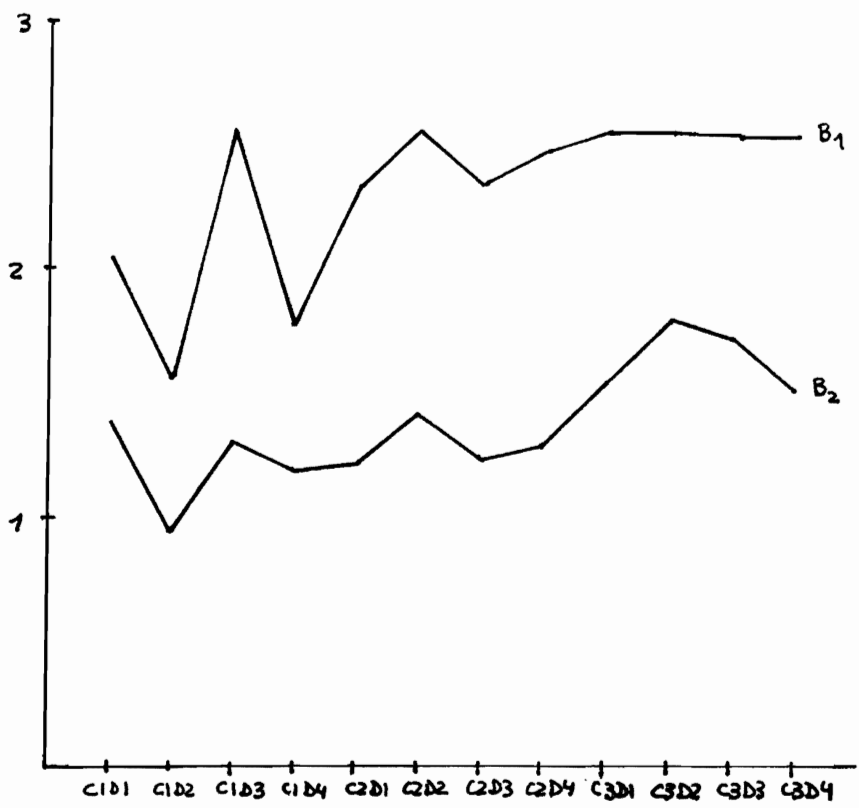

Por otro lado, efectuamos comparaciones de interacción entre las tareas tomadas dos a dos y agrupando las de conmutatividad frente a la tarea de resolver sumas, en cada uno de los sumandos. Los resultados muestran diferencias significativas tan sólo en el sumando hechos numéricos superiores a la decena entre las tareas resolver sumas-comparar sumas $\left(\mathrm{F}_{1,414}=8.002, \mathrm{p}<0.01\right)$, resolver sumas-encontrar el sumando desconocido $\left(\mathrm{F}_{1,444^{-5}} .23, \mathrm{p}<0.05\right)$ y resolver sumas-tareas de conmutatividad consideradas conjuntamente $\left(\mathrm{F}_{1,414}-8.78, \mathrm{p}<0.01\right)$.

Resumiendo, el factor presencia del resultado afecta al nivel de éxito de los sujetos en las tres tareas y en todos los sumandos. A este respecto, la competencia de los niños tanto en la suma como en la propiedad conmutativa tiene lugar primeramente en situaciones en las que el resultado no se encuentra presente. Este descenso en el nivel de rendimiento en las tres tareas cuando el resultado se halla presente puede relacionarse con el desconocimiento por parte de los sujetos de la función del signo igual. Numerosos estudios (Baroody y Ginsburg, 1986; Behr, Erlwanger y Nichols, 1976; Kieran, 1981; etc.) apuntan que el fracaso de los niños en algunas tareas de adición se debe a que otorgan a este símbolo matemático un sentido de operador, es decir, se interpreta como un signo de «suman» o «hacen un total de...». En el presente estudio tal desconocimiento se evidencia en los errores cometidos por los niños en las tres tareas, como veremos más adelante en el apartado correspondiente al análisis de los errores.

\section{Análisis de las relaciones entre los factores presencia/ausencia del resultado, grupo y sumandos}

Por último, en lo que se refiere a la interacción presencia del resultado $\mathrm{x}$ grupo $\mathrm{x}$ tipo de sumandos, en un nivel más general, llevamos a cabo comparaciones de inte- 
racción entre los distintos nivẹles del factor grupo, asignando el mismo peso a los sumandos, en el factor presencia/ausencia del resultado. Los resultados muestran diferencias significativas entre los grupos I-III $\left(\mathrm{F}_{1,207}=28.71, \mathrm{p}<0.01\right)$ y II-III $\left(\mathrm{F}_{1,207}\right.$ $=26.9$, $\mathrm{p}<0.01$ ), pero no entre los grupos I y II.

Por otra parte, el análisis de las comparaciones de interacción entre los grupos en cada uno de los sumandos, en el factor presencia/ausencia del resultado, revela que existen diferencias significativas entre los grupos I y III $\left(\mathrm{F}_{1,207}=7.04, \mathrm{p}<0.01\right)$, II y $\operatorname{III}\left(\mathrm{F}_{1,207}=9.02, \mathrm{p}<0.01\right)$ en el sumando $1+\mathrm{N}$. Asimismo llegan a ser significativas las comparaciones entre los grupos I y III $\left(\mathrm{F}_{1,207}=14.89, \mathrm{p}<0.01\right)$ en el sumando círculos + guarismos, entre los grupos II y III $\left(\mathrm{F}_{1,207}=9.33, \mathrm{p}<0.01\right)$ en el sumando hechos numéricos y, finalmente, entre los grupos I y III $\left(\mathrm{F}_{1,207}=4.71, \mathrm{p}<0.01\right)$ y entre los grupos II y III $\left(\mathrm{F}_{1,207}=10.86, \mathrm{p}<0.01\right)$ en el sumando hechos numéricos superiores a la decena.

En síntesis y de acuerdo con nuestra hipótesis (1), existen diferencias evolutivas entre los grupos I y II respecto al grupo III, en el factor presencia/ausencia del resultado y estas diferencias se evidencian en los sumandos $1+\mathrm{N}$, círculos + guarismos y hechos numéricos.

\section{ANALISIS DE LAS ESTRATEGIAS DE SOLUCION}

\section{Las estrategias aditivas}

Las estrategias utilizadas por.los niños, cuando la incógnita se ubica en el resultado, son de tres tipos: modelado directo, conteo y hechos numéricos, existiendo diferencias evolutivas entre los grupos. En concreto, en el grupo de preescolar las estrategias más habituales son las de modelado directo $(32,64 \%$ de los ensayos) y conteo (61,54\% de los ensayos). En $1 .^{\circ}$ de EGB sobresalen las estrategias de conteo (53,8\% de los ensayos) y en un segundo plano las de hechos numéricos $(33,09 \%$ de los ensayos). Finalmente, en $2 .^{\circ}$ de EGB las estrategias de hechos numéricos (48,46\% de los ensayos) alcanzan un porcentaje ligeramente superior a las de conteo ( $46,77 \%$ de los ensayos). Finalmente, hallamos distintos niveles de evolución en las estrategias de modelado directo y conteo, que han sido recogidas ampliamente en un trabajo anterior (Bermejo y Rodríguez, en prensa), a donde remitimos al lector interesado.

Cuando la incógnita se ubica en el sumando inicial, los niños utilizan preferentemente la estrategia de contar desde el sumando conocido hasta el resultado. En concreto, esta estrategia se presenta en el $70,92 \%$ de los ensayos en el grupo de preescolar, en el $60,94 \%$ de $1 .^{\circ}$ de EGB y en el $53,12 \%$ en $2 .^{\circ}$ de EGB. Sin embargo, tan sólo en el 10,61\% de los ensayos de preescolar, en el 0,89\% de los de $1 .^{\circ}$ de EGB y en el $2,23 \%$ de los de $2 .^{\circ}$ de EGB usan la estrategia de contar hacia atrás relacionada con la resta.

Por último, las estrategias de hechos numéricos aparecen escasamente en los grupos de preescolar y $1 .^{\circ}$ de EGB, mientras que cobran especial importancia en $2 .^{\circ}$ de $\operatorname{EGB}(39,38 \%$ de los ensayos).

\section{Las estrategias en las tareas de conmutatividad}

En la tarea de comparar sumas, cuando el resultado no se halla presente, encontramos cuatro tipos de estrategias: (1) aquellas que se centran en los sumandos (los sumandos están al revés, los dos sumandos son iguales, uno de los sumandos es igual), (2) las que aluden a que el resultado de ambas operaciones es el mismo (solucionan ambas cuentas, una de ellas, ninguna de las dos), (3) las que hacen mención directamente a la propiedad conmutativa y (4) las que señalan que tanto los suman- 
dos como los signos son los mismos en una y otra operaciones (Tabla II). En presencia del resultado, se manifiestan básicamente las mismas estrategias.

Tabla II

Estrategias utilizadas en las tareas de conmutatividad en ausencia/presencia del resultado. Porcentajes de ensayos correspondientes a cada estrategia

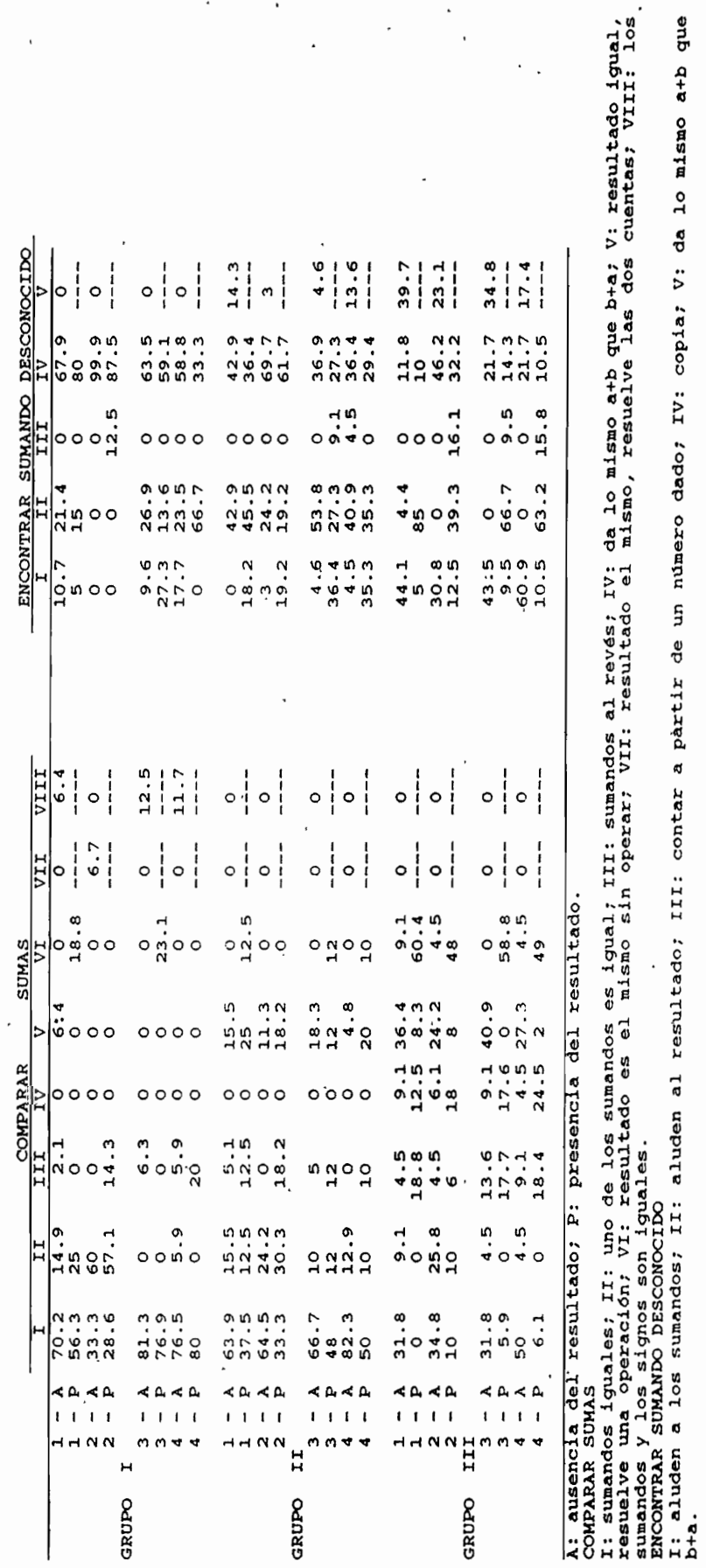


El análisis de estos datos muestran que, tanto en presencia como en ausencia del resultado y en contra del estudio de Baroody y Gannon (1984), los niños de los grupos I y II se centran principalmente en los sumandos a la hora de determinar si dos operaciones aditivas son o no equivalentes, independientemente del tipo de sumando de que se trate. No obstante, es posible que algunos preescolares no posean un conocimiento suficientemente elaborado de la propiedad conmutativa y se trate más bien de una comparación entre las operaciones a un nivel estrictamente perceptivo, de modo que no aludan a la equivalencia de las adiciones, sino más bien a la equivalencia de los cardinales que representan los sumandos. Así parece confirmarlo, en parte, el hecho de que de los 16 sujetos del G. I que resuelven correctamente la tarea de comparar sumas en el sumando «hechos numéricos» en ausencia del resultado, 9 de ellos necesitan sumar en las dos cuentas para obtener el resultado; mientras que en el G. II lo hacen 7 de los 20 sujetos y țan sólo 2 de 22 en el G. III. En este último grupo aumenta considerablemente la estrategia que se refiere al resultado. Ello se debe a que los niños poseen un mayor conocimiento conceptual y de procedimiento de la adición, poniendo en marcha procedimientos memorísticos. Así, cuando emiten el juicio de conmutatividad en la tarea de comparar sumas aluden a los sumandos o al resultado, dependiendo de que conozcan previamente o no el resultado de ambas operaciones.

En la tarea de encontrar el sumando desconocido, en ausencia del resultado, las estrategias son de cinco tipos: (1) las que se centran en los sumandos («porque aquí hay... y aquí no»), (2) las que lo hacen en el resultado ("porque a + b son c y para tener igual aquí es $b »)$, (3) aquellas que aluden directamente a la propiedad conmutativa ( da lo mismo decir $\mathrm{a}+\mathrm{b}$ que $\mathrm{b}+\mathrm{a}$, porque el resultado es el mismo»), (4) las consistentes en copiar simplemente el número que falta mirando en la otra cuenta ( «lo he visto aquí», «lo pone aquí») y, finalmente, (5) la estrategia aditiva de contar a partir de un número dado (i.e., resuelven la cuenta en la que figuran los dos sumandos anotando el resultado en la otra y, seguidamente, cuentan en esta última desde el sumando conocido hasta el resultado) ('Tabla II). Cuando el resultado está presente se mantienen las mismas estrategias, exceptuando la que alude directamente a la conmutatividad.

Los datos procedentes de esta prueba, tanto si el resultado está presente como si está ausente, indican que los niños del G. I encuentran el sumando desconocido copiándolo simplemente de la otra cuenta. En este grupo de los 15 .sujetos que ejecutan correctamente las dos tareas de conmutatividad en ausencia del resultado, bien mediante el procedimiento de copia en la tarea de encontrar el sumando desconocido, bien aludiendo a los sumandos en la tarea de comparar sumas, 7 de ellos no necesitan sumar en esta última tarea en ambas cuentas en el sumando hechos numéricos. Este resultado sugiere que algunos sujetos de este grupo poseen la propiedad conmutativa y, por tanto, lo que puede haber ocurrido en la tarea de encontrar el sumando desconocido es que sufren una cierta regresión, debido simplemente a que se trata de una tarea más sencilla.

En el G. II, aunque se mantiene esta pauta general de resultados, una buena parte de los sujetos hacen mención del procedimiento referido al resultado en ambas condiciones. Además, algunos sujetos (i.e., 8) que utilizan la estrategia consistente en afirmar que los sumandos son iguales en la tarea de comparar sumas, saben también que el resultado de ambas cuentas es el mismo, puesto que todos ellos aluden al resultado en la tarea de encontrar el sumando desconocido y ninguno necesita realizar las dos cuentas en la prueba de comparar sumas en el sumando hechos numéricos. Algo semejante puede afirmarse en relación con el G. III, ya que tanto en esta tarea como en la de comparar sumas, ponen en marcha estrategias relativas a los sumandos o el resultado.

\section{Las estrategias aditivas y las tareas de conmutatividad}

La hipótesis 3 proponía que la utilización de estrategias aditivas consistentes en contar empezando por el sumando mayor supone una cierta competencia por parte 
TABLA III

Porcentajes de ensayos correspondientes a los éxitos/fracasos en algunas estrategias aditivas en las tareas de conmutatividad

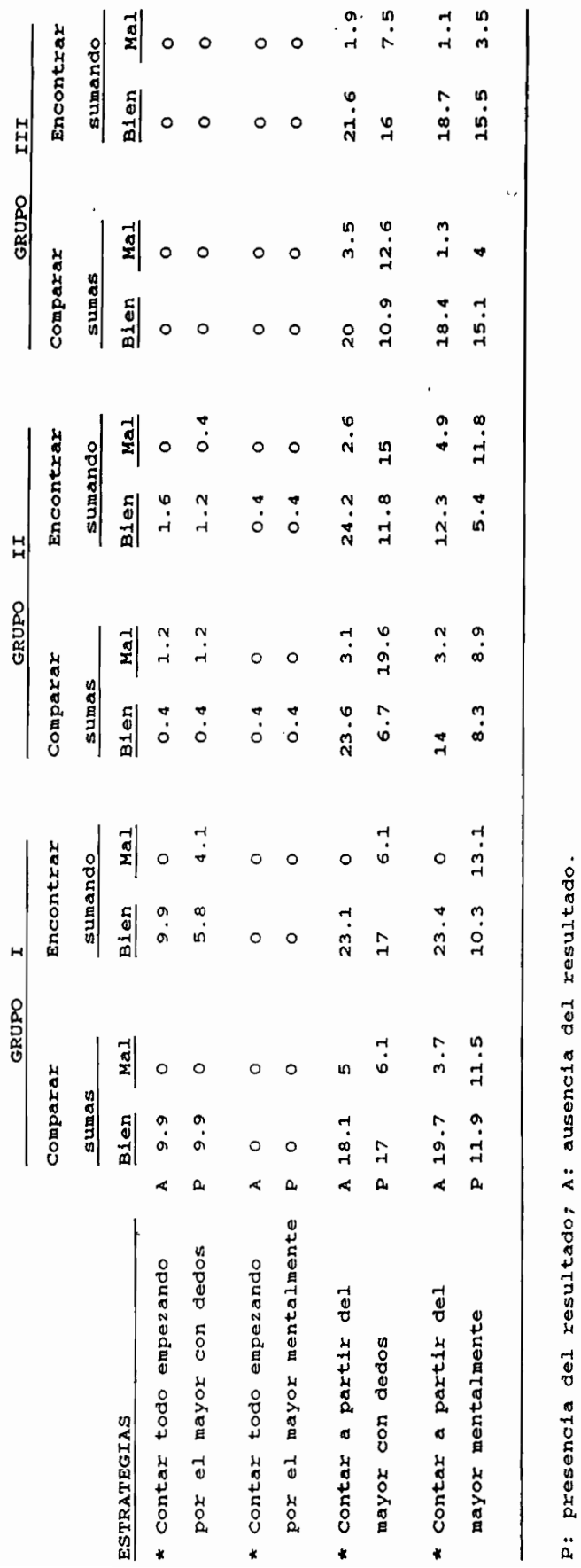


del niño con respecto a la propiedad conmutativa. Los resultados (Tabla III) indican que los niños preescolares poseen una cierta comprensión del principio de conmutatividad, como lo demuestra el hecho de que el porcentaje de éxitos es superior al de fracaso en las tareas de conmutatividad en las estrategias de contar todo con los dedos empezando por el mayor y contar a partir del mayor con los dedos. En la estrategia de contar a partir del mayor mentalmente, se mantiene este resultado en la tarea de comparar sumas y encontrar el sumando desconocido en ausencia del resultado, pero no en presencia del mismo. En $1 .^{\circ}$ de EGB el porcentaje de ensayos correctos supera al de fracasos en las tareas de conmutatividad en la estrategia de contar todo mentalmente empezando por el mayor. En las tareas de comparar sumas y encontrar el sumando desconocido en ausencia del resultado, los éxitos son superiores a los fracasos únicamente en las estrategias de contar a partir del mayor con los dedos y contar a partir del mayor mentalmente. Finalmente, en el G. III abundan los éxitos en la estrátegia de contar a partir del mayor mentalmente y con los dedos, aunque en este último caso hay que exceptuar la tarea de comparar sumas con el resultado.

Estos datos sugieren dos interpretaciones complementarias. En primer lugar, de acuerdo con nuestra tercera hipótesis y en contra de otros autores (Baroodý y Ginsburg, 1986; Resnick y Neches, 1984), los datos del presente estudio indican que la utilización por parte del niño de las estrategias consistentes en contar todo empezando por el mayor y contar a partir del mayor conllevan siempre un cierto conocimiento de la propiedad conmutativa. En segundo lugar, el conocimiento incompleto de la conmutatividad es lo que puede propiciar que, al menos en los grupos I y II, además de la estrategia de contar empezando por el mayor, los niños recurran a otros procedimientos de ejecución menos elaborados en las tareas aditivas. En otras palabras, la regresión que algunos autores (Carpenter, 1986; Carpenter y Moser, 1982, 1984) señalan en los procedimientos de resolución empleados por los niños en las tareas aditivas puede ser debida entre otras cosas al conocimiento incompleto del principio de conmutatividad.

\section{ANALISIS DE ERRORES}

\section{Los errores en la tarea de sumar}

Tomando como referencia el modelo de conteo de Greeno, Riley y Gelman (1984), establecemos tres tipos de errores: (a) de competencia conceptual, (b) de competencia de procedimiento y (c) de competencia de utilización. Los primeros se relacionan con un conocimiento incompleto de los principios y reglas subyacentes a la operación de sumar (i.e., inventar la respuesta, repetir una de las cantidades ya dadas, etc.). Los errores de competencia de procedimiento se producen porque los niños seleccionan estrategias inadecuadas para llevar a buen término la tarea (i.e., intentar representar los dos sumandos con los dedos cuando no se posee el número suficiente de dedos). Por último, los errores relativos a la competencia de utilización implican competencia conceptual y de procedimiento por parte del niño, pero fallan en la puesta en marcha del procedimiento seleccionado (i.e., contar mal) (para más información, Bermejo y Rodríguez, en prensa; Rodríguez, en prensa).

Teniendo en cuenta esta categorización de los errores, podemos observar que en las tareas con la incógnita en el resultado son frecuentes los errores conceptuales entre los niños de preescolar (57,46\% de los ensayos) y $1 .^{\circ}$ de $\operatorname{EGB}(56,07 \%$ de los ensayos), consistiendo fundamentalmente en la repetición de una de las cantidades. Sin embargo, los niños mayores únicamente cometen un error de este tipo en la tarea círculos + guarismo. Los errores de procedimiento aparecen solamente en el grupo de los más pequeños (24,49\% de los ensayos), mientras que los de utilización se 
manifiestan en los tres grupos (18,04\% de los ensayos en preescolar, $43,92 \%$ en $1 .^{\circ}$ de EGB y $90 \%$ en $2 .^{\circ}$ de EGB).

Cuando la incógnita se ubica en el sumando inicial, constatamos que los errores más frecuentes en todos los grupos y en todas las tareas. son de tipo conceptual $\left(86,67 \%\right.$ de los ensayos en preescolar, $85,65 \%$ en $1 .^{\circ}$ de EGB y $64,22 \%$ en $2 .^{\circ}$ de EGB), siendo no obstante menos numerosos en $2 .^{\circ}$ de EGB. Los errores de procedimiento y utilización ( $15,45 \%$ de los ensayos y $20,33 \%$, respectivamente) son también frecuentes en este último grupo.

\section{Los errores en las tareas de conmutatividad}

En là prueba de comparar șumas en ausencia del resultado se presentan dos categorías de errores: (1) afirmar que no existe la misma cantidad porque los sumandos están al revés y (2) afirmar la igualdad sustituyendo el primer sumando de la segunda cuenta por un número cualquiera en su lugar. Los errores más frecuentes en todos los grupos corresponden a la primera categoría mencionada (Tabla IV).

En presencia del resultado, los errores son de 5 tipos: (1) errores de tipo perceptivo consistentes en indicar que falta el resultado o que falta el resultado y el signo de igualdad, (2) errores que inciden en que el resultado de ambas operaciones es distinto, (3) errores referentes a que los sumandos están al revés, (4) errores mixtos en los que los niños argumentan que en úna de las cuentas falta el resultado y los sumandos están al revés y (5) errorés no categorizables. El mayor porcentaje de ensayos incorrectos en todos los grupos, así como en los cuatro tipos de sumandos, corresponde al primer tipo de errores que hemos considerado, ya que, bien no admiten la igualdad porque en uña de las cuentas aparece el resultado mientras que en la otra no, bien indican que en una de las operaciones no figura el signo igual ni tampoco el resultado.

En ausencia del resultado, los errores se agrupan en tres categorías: (1) repetición de cantidades, (2) las consistentes en resolver la cuenta en la que se consignan ambos sumandos anotando el resultado en el lugar correspondiente al sumando desconocido y (3) no categorizables. Entre los más pequeños los errores más frecuentes corresponden en todos los sumandos a la repetición de cantidades. Estos errores, que son semejantes a los que se producen en la tarea de sumar cuando la incógnita se ubica en el primer sumando, indican que los niños se centran en los sumandos y, dado que éstos se encuentran al revés y el resultado de las cuentas debe ser el mismo, ofrecen como respuesta el sumando conocido o escriben la operación sobre la que se realizan las comparaciones (Tabla IV).

En $1 .^{\circ}$ y $2 .^{\circ}$ de EGB predominan los errores consistentes en resolver la operación en la que los sumandos son conocidos, situando el resultado en la segunda operación en el lugar correspondiente al sumando desconocido. Estos errores ponen de manifiesto que los niños no disponen de conocimiento referente al valor de las cantidades según se encuentren antes del signo de igualdad o después del mismo.

Cuando el resultado está presente, se observan las siguientes categorías de estrategias incorrectas: (1) ofrecer como respuesta el resultado de la primera cuenta con o sin el signo igual, (2) indicar correctamente el sumando desconocido, pero sin admitir la igualdad de los algoritmos, bien porque el orden de los sumandos es distinto, bien porque en uno de ellos no figura el resultado, (3) dar como respuesta la misma cantidad que el sumando conocido, (4) poner una cantidad distinta a cualquiera de las existentes en los algoritmos, (5) considerar que el sumando desconocido lo constituye la suma del resultado con el sumando inicial en la primera operación, (6) equiparar el sumando desconocido con la primera operación ya sea con o sin el resultado y (7) consignar como respuesta el resultado del primer algoritmo, acompañándolo de alguno de los sumandos y, en ocasiones; además del signo igual. 
TABLA IV

Porcentajes de ensayos en los distintos tipos de errores en las tareas de conmutatividad en ausencialpresencia del resultado

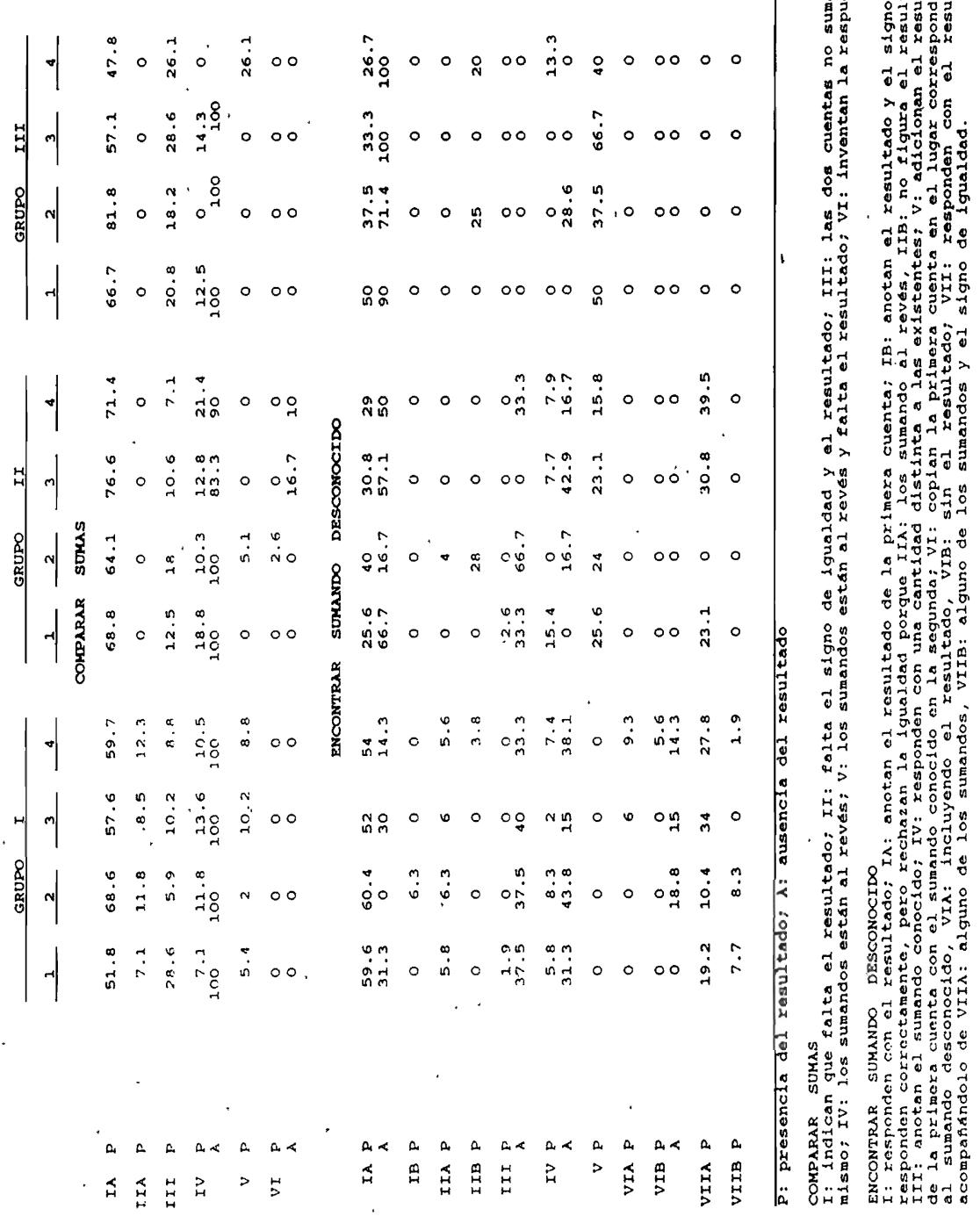


Los errores más frecuentes en preescolar y $1 .^{\circ}$ de EGB (Tabla IV) consisten en consignar como respuesta el resultado en el lugar del sumando desconocido. En el grupo de los mayores, aunque persisten estos errores, son también frecuentes los que consisten en sumar en la primera operación el sumando inicial con el resultado de la misma, anotando a continuación este resultado en el lugar del sumando desconocido.

En general, los errores cometidos por los niños a lo largo de esta prueba se pueden atribuir, por un lado, a que no identifican correctamente o bien significado de las cantidades, indicando como respuesta el resultado, o bien el valor de los signos, de modo que a menudo los niños anotan además como respuesta el signo de igualdad. Por otro lado, aunque en un porcentaje más bien bajo, algunos sujetos escriben como respuesta el primer algoritmo, lo que desde nuestro punto de vista puede tener dos interpretaciones posibles: bien, de acuerdo con la explicación citada más arriba, no conocen claramente el significado de los términos aditivos, bien porque el sumando conocido no se sitúa en el lugar adecuado, intentando eliminar esta dificultad escribiendo toda la operación de nuevo.

\section{Los errores aditivos y las tareas de conmutatividad}

En este apartado consideramos las respuestas erróneas de los niños en la tarea de sumar, tanto en presencia como en ausencia del resultado, y su relación con el éxito o fracaso en las tareas de conmutatividad. Como podemos observar en la Tabla V, en todos los grupos, los errores aditivos que se producen cuando la incógnita se sitúa en el resultado se acompañan de un porcentaje mayor de fracaso en las tareas de comparar sumas y encontrar el sumando desconocido cuando el resultado se encuentra presente en estas últimas. Sin embargo, esta pauta de resultados no se repite en ninguno de los grupos cuando en esas mismas tareas está ausente el resultado. En suma, los datos aquí encontrados ponen de manifiesto que antes de que los niños presenten una cierta maestría en la operación aditiva, poseen ya una cierta competencia con respecto a la conmutatividad. Es decir, de acuerdo con la hipótesis 4 , los ensayos incorrectos en las tareas de sumar no siempre se corresponden con los ensayos incorrectos en las tareas de conmutatividad, principalmente cuando en éstas se halla ausente el resultado.

En la tarea de sumar con la incógnita en el sumando inicial (Tabla V) se producen resultados similares. Es decir, en todos los grupos y en ambas tareas de conmutatividad en ausencia del resultado, el éxito supera al fracaso. Sin embargo, en las tareas de conmutatividad en presencia del resultado, aunque en los grupos de $10^{\circ}$ y $2 .^{\circ}$ de EGB el éxito sigue siendo superior al fracaso con respecto a los errores de procedimiento y utilización, no ocurre esto mismo en concreto en los errores conceptuales, en donde la relación se invierte. Estos datos están en desacuerdo con las afirmaciones de Weaver (1982) cuando supone que tanto la resolución correcta de las tareas de adición con la incógnita en el sumando inicial como la adquisición de la propiedad conmutativa comportan un esquema de adición binario. Nuestros resultados muestran un fracaso considerable en la tarea de sumar con la incógnita en el sumando inicial, sobre todo en los grupos I y II, pero no encontramos un fracaso similar en las tareas de conmutatividad, en ausencia del resultado y en algunas ocasiones en presencia del mismo (errores de procedimiento y utilización). Por tanto, cabrían al menos dos interpretaciones posibles, o bien la adquisición de la propiedad conmutativa se asocia primeramente con un esquema unitario de la adición, o bien estos sujetos se hallan en un período de transición hacia la concepción binaria de la adición, pero debido a la dificultad de esta tarea de sumar, no manifestarían dicho conocimiento en relación con la adición, pero sí en algunas de las tareas conmutativas. 
TABLA V

Errores en la tarea de sumar con la incógnita en el resultado y sumando inicial. Porcentajes de ensayos correspondientes a los éxitos/fracasos en las tareas de conmutatividad en ausencia y presencia del resultado

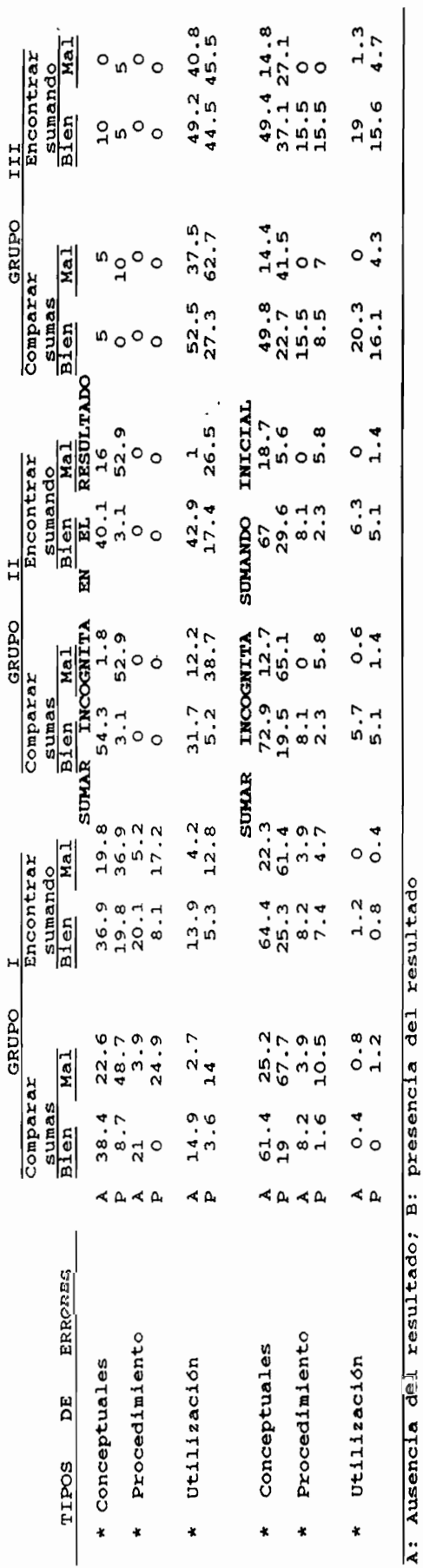




\section{CONCLUSIONES}

Los distintos factores estudiados (edad, presencia/ausencia del resultado, tipo de tarea y tipo de sumandos) parecen ejercer una influencia de manera aislada o en interacción sobre el rendimiento de los niños. Más concretamente, a medida que ascendemos en el nivel de escolaridad tanto la resolución de la tarea de sumar como la aplicación de la propiedad conmutativa se hace de forma más sistemática a lo largo de las distintas condiciones experimentales. Igualmente, nuestros datos ponen de manifiesto que en presencia del resultado el éxito de los niños resulta inferior, en general, en todas las tareas. Además, el factor tipo de sumandos, tomado aisladamente, si bien no parece influir sustancialmente en el rendimiento de los niños en las tareas de conmutatividad, sí lo hace en la tarea de sumar. En esta tarea, el rendimiento resulta inferior en los sumandos círculos + guarismo y hechos numéricos superiores a la decena.

Por lo que se refiere a las estrategias de solución, en la tarea de resolver; sumas, la presencia/ausencia del resultado, el tipo de sumandos y el grupo determinan el procedimiento seleccionado por los niños. Sin embargo, en las tareas de conmutatividad se producen diferencias en las estrategias en función de la presencia/ausencia del resultado, pero no en relación con los factores tipo de tarea y tipo de sumandos. Además, en estas tareas el análisis de las estrategias pone de manifiesto la existencia de diferencias cualitativas importantes entre ellas, que pueden implicar distintos grados de conocimiento de la propiedad conmutativa.

Por otro lado, nuestros datos revelan en contra de otros autores (Baroody y Gannon, 1984; Resnick y Neches, 1984) que la utilización de estrategias aditivas consistentes en contar empezando por el sumando mayor se acompaña siempre de un cierto éxito en las tareas de conmutatividad. Exito que cada vez resulta superior a medida que aumenta el nivel de escolaridad de los niños. Estos resultados pueden ser interpretados, de acuerdo con Carpenter, en el sentido de que el conocimiento de procedimiento no se produce al margen del conocimiento conceptual o, en otras palabras, el procedimiento de contar a partir del mayor no puede desvincularse de un cierto conocimiento de la propiedad conmutativa. Desde nuestro punto de vista, al igual que los procedimientos presentan distintos niveles de complejidad, los conceptos presentan igualmente distintos grados de elaboración. En esta línea, los datos del presente estudio muestran que la adquisión de la propiedad conmutativa de la suma se produce de manera gradual. Baste recordar que las estrategias más completas y elaboradas, como la consistente en afirmar la equivalencia aludiendo a que el resultado de los dos algoritmos es equivalente sin necesidad de resolverlos, corresponden a los niños mayores, mientras que las más sencillas (i.e., perceptivas) aparecen entre los niños más pequeños. En la tarea de sumar se produce un fenómeno similar, ya que, por ejemplo, la estrategia de contar a partir del sumando mayor gana en consistencia a medida que el nivel de escolaridad es mayor. A este respecto, estamos en desacuerdo con Baroody y Gannon (1984) cuando afirman que la estrategia de contar a partir del mayor deriva de un proceso de economía cognitiva. En caso de que esto fuera así, cabría esperar que una vez descubierta esta estrategia por los niños la utilizarían siempre en detrimento de otras más sencillas, como contar todo. Sin embargo, numerosos estudios (i.e., Bermejo, 1990; Bermejo y Rodríguez, 1987) encuentran datos contrarios a esta suposición. Por tanto, esto nos lleva a concluir que el conocimiento incompleto de la propiedad conmutativa de la adición, que en un principio tienen los niños, explicaría que los niños recurran a estrategias aditivas más sencillas aun conociendo otras más complejas.

Finalmente, en cuanto a los errores, los factores presencia/ausencia del resultado y tipo de tarea inciden en la aparición de distintos tipos de errores, pero no el factor tipo de sumandos. Además, la resolución incorrecta de las tareas aditivas no se acompaña siempre de fracaso igualmente en las tareas de conmutatividad. Por tanto, la adquisición de la propiedad conmutativa corre paralela al desarrollo del concepto de 
adición. En otras palabras, antes de que los niños hayan alcanzado un concepto completo y elaborado de la adición, que en términos de Weaver (1982) supondría la posesión de la concepción binaria de la suma, poseerían ya un cierto conocimiento de la propiedad conmutativa.

\section{Referencias}

BAROODY, A. J., y GANNON, K. E. (1984) . The development of the commutativity principle and economical addition strategies. Cognition and Instruction, 1, 321-339.

BAROODY, A. J., y GINSBURG, H. P. (1986). The relationships between initial meaningful and mechanical knowledge of arithmetic. En J. Hiebert (Ed.), Conceptual and procedural knowledge: The case of mathematics (pp. 75-112). Hillsdale, NJ: LEA.

Baroody, A. J.; GinsBurg,H. P., y WaXman, B. (1983). Children's use of mathematical structure. Journal for Research in Mathematics Education, 14, 156-168.

BeHR, M.; ERLWANGER, S., y Nichols, E. (1976). How children view equality sentences (PMDC Technical Report N. ${ }^{\circ}$ 3). Tallahassee: Florida State University. Citado por Kieran, C. (1981).

Bermejo, V. (1990). El niño y la aritmética. Barcelona: Paidós.

BERMEJO, V., y RODRf́GUEZ, P. (1987). Estructura semántica y estrategias infantiles en la solución de problemas verbales de adición. Infancia y Aprendizaje, 39-40, 71-81.

BERMEjo, V., y Rodríguez, P. (en prensa). Conceptualización de la operación aditiva y estrategias de solución. Investigaciones Psicológicas.

BYRNES, J. P., y W ASIK, B. A. (1991). Role of conceptual knowledge in mathematical procedural learning. Developmental Psychology, 27 (5), 777-786.

BRIARS, D. J., y LARKIN, J. H. (1984). An integrated model of skills in solving elementary word problems. Cognition and Instruction, 1 245-296.

CARPENTER, T. P. (1986). Conceptual knowledge as a foundation for procedural knowledge: Implications from research on the initial learning of arithmetic. En J. Hiebert (Ed.), Conceptual and procedural knowledge: The case of mathematics (pp. 113-132). Hillsdale, NJ: LEA.

CARPENTER, T. P., y MOSER, J. M. (1982). The development of addition and subtraction problem-solving skills. En T. P. Carpenter, J. M. Moser y T. Romberg (Eds.), Addition and subtraction: A cognitive perspective (pp. 9-24). Hillsdale, NJ: LEA.

CARPENTER, T. P., y MOSER, J. M. (1984). The acquisition of addition and subtraction concepts in grades one through three. Joumal of Research in Mathematics Education, 15, 179-202.

GreENO, J. G.; RILEY, M. S., y GeLMAN, R. (1984). Conceptual competence and children's counting. Cognitive Psychology, 16, 94-143.

HieberT, J., y LeFEVRE, P. (1986). Conceptual an procedural knowledge in mathematics: An introductory analysis. En J. Hiebert (Ed.), Conceptual and procedural knowledge: The case of mathematics (pp. 129). Hillsdale, NJ: LEA.

KIERAN, C. (1981). Concepts associated with the equality symbol. Educational Studies in Mathematics, 12, 317-325.

Miller, K.; Keating, D., y Perlmutter, M. (1984). Cognitive arithmetic: Comparison of operations. Journal of Experimental Psychology: Learning, memory, and cognition, 10, 46-60.

RESNICK, L. B. (1983). A developmental theory of number understanding. En H. Ginsburg (comp.), The development of mathematical thinking (pp. 136-155), Nueva York, Academic Press.

RESNICK, L. B., y NECHES, R. (1984). Factors affecting individual differences in learning ability. En R. J. Sternberg (Ed.), Advances in the psychology of buman intelligence (pp. 275-323). Hillsdale, NJ: LEA.

RILEY, M. S.; Greeno, J. G., y HeLleR, J. I. (1983). Development of children's problem-solving ability in arithmetic. En H. Ginsburg (Ed.), The development of mathematical thinking (pp. 153-196). Nueva York: Academic Press.

Rodríguez, P. (en prensa). Análisis de los procesos cognitivos que conducen a la adquisición de la propiedad conmutativa. Madrid: Universidad Complutense.

SIIVER, E. A. (1986). Using conceptual and procedural knowledge: A focus on relationships. En J. Hiebert (Ed.), Conceptual and procedural knowledge: The case of matbematics (pp. 181-198). Hillsdale, NJ: LEA.

VANLEHN, K. (1986). Arithmetic procedures are induced from examples. En J. Hiebert (Ed.), Conceptual and procedural knowledge: The case of mathematics (pp. 133-180). Hillsdale, NJ: LEA.

WEAVER, J. F (1982). Interpretations of number operations and symbolic representations of addition and subtraction. En T. Carpenter, J. Moser y T. Romberg (Eds.), Addition and subtraction: A cognitive perspective (pp. 60-66). Hillsdale, NJ: LEA. 